\title{
FROM CAD TO PHYSICS-BASED DIGITAL TWIN: FRAMEWORK FOR REAL-TIME SIMULATION OF VIRTUAL PROTOTYPES
}

\author{
J. G. Pereira and A. Ellman $\bowtie$ \\ Tampere University, Finland \\ $\triangle$ asko.ellman@tuni.fi
}

\begin{abstract}
Engineering work is mostly done in 3D CAD software throughout the engineering process from conceptual design and layout of products. Physics-Based Virtual Prototypes are very valuable addition on Computer Aided Engineering enabling product development simulators, training simulators and digital twin concept in product lift-cycle process. In this work, we present a framework, how such virtual prototypes can be developed from 3D CAD models with meaningful effort.
\end{abstract}

Keywords: 3D modelling, virtual prototyping, virtual reality (VR)

\section{Introduction}

In product development process, engineering work is mostly done in 3D CAD software throughout the engineering process from conceptual design and layout of products, through strength and dynamic analysis of assemblies to definition of manufacturing methods of components. These 3D CAD models are commonly visualized, tested and evaluated via desktop interface. In recent years, Virtual Reality (VR) technology has become widely available as a development tool for the industry field. One of the main advantages of VR is the possibility to see virtual prototypes in real scale and from various perspective. Reviewing a model with higher complexity using a VR system might be beneficial for better understanding the product (Horvat et al., 2019). This makes possible for stakeholders with nontechnical background participate in the design process, since they may not be able to read technical blueprints (Tiainen et al., 2014).

Nowadays VR and Augmented Reality (AR) systems offer great potential for business usage. It enables the conduction of aesthetic, ergonomic or other design evaluations at early design stages. This can lead to a significant reduction of development time, because the product developers can perform fast and simple virtual variant analysis instead of building and executing costly (and sometimes dangerous) experiments with physical prototypes. The designer may get intuitive access to various mechanical components, which is particularly advantageous for design modifications with existing digital models, contributing to cost savings and sustainable development. During a decade, VR technology has developed rapidly, resulting in low operational cost and fast process time (Ellman and Tiainen, 2019). This immersive technology has been adopted by many industries in their workflow, because of the advantages that they offer to design practices (Berg and Vance, 2017).

Game engines, as Unity, are increasingly explored for design applications. They offer good support to VR/AR devices such as Oculus Rift and Microsoft HoloLens, which makes their use very appealing. 
However, introducing parametric CAD models to game engines is still an issue due to the technology specifications and high time consuming (Ekströmer et al., 2019), often requiring the involvement of information technology specialists. Moreover, so called game physics are based on simplified calculation of momentum, which is inadequate if more exact results are needed.

A mobile work machine is a challenging application for virtual prototyping. The fact that such entities consist of machine mechanisms, mechanical, hydraulic and/or electronic powertrains, results in a complex simulation model. However, it is possible to achieve real-time simulation if special attention is paid on modelling (Esqué et al., 2003). By introducing a mechanical model with real motion equation and using special algorithms (Lacoursière, 2007), a physics-based real-time simulator can be reached, which is capable to provide very realistic machine behaviour.

Physics-based real-time virtual prototype of mobile work machine enables several possibilities to its product lifecycle. Firstly, can be used as product development simulator at design phase. Secondly, it can be altered to training simulator for teaching future users of the product in use phase. Thirdly, is can be altered to digital twin of the product to be used for monitoring the machine operation during lifecycle. Digital twin enables access to use of the individual machines and therefore it provides a new feedback to design process (Grieves and Vickers, 2017). Traditionally implementation of such entities has been a major effort. Therefore, this paper proposes the following research questions:

- Is there a systematic framework for implementation of physics-based virtual prototypes from 3D CAD models?

- How large effort does implementation of such model take?

This work presents a detailed approach of complex virtual prototypes in virtual environments (VE), enabling further opportunities throughout product lifecycle in the early stages of development. Over different software platforms, a general modular framework was established and implemented in a case study of a harvester CAD model. The case study shows and discusses the conversion of the model into a physics-based real-time simulation environment.

\section{Physics-based virtual prototypes}

Product lifecycle consists of various phases which results in diverse needs for design software. Engineering work is mostly done in 3D CAD software and CAD software companies, such as Siemens and Dassault Systèmes, try to fulfil those needs by set of simulation software which can exchange design information. Many of product lifecycle needs, as user training and condition monitoring, are not currently covered in this software. However, several products, as large mobile work machines with complex control and actuator system, require training simulators to teach new product users. The training simulator is usually a separate product that is presumed by the machine manufacturer. The digital twin concept has emerged a new need for monitoring product use and makes it possible to offer condition monitoring services to machine owners.

Verifying requirements related to dynamic properties of the machine can be challenging. This is especially true for mobile machines. Product development simulators with high-fidelity simulation models have become popular for solving this issue.

The product simulation software has recently developed to a direction where it offers several interfaces for various purposes. This feature can make it possible to create modular software entities. It also may include a model library for certain domains. However, parameter data must be identified by the user. Consequently, a CAD software can offer structure of mechanics model and most of the parameters for the dynamic simulation of a mechanical structure. Therefore, it is a valuable feature if a CAD model can be used in the construction of a mechanical model.

The VE can be considered as a separate software entity. It contains means for interfacing, and it provides a certain level of immersion. The VE software has support for several physical input and output devices. If a real-time physics-based simulation model can be combined with a VE software, a real-time physics-based virtual prototype is achieved. This kind of simulator is very useful in product development, as it enables the participation of non-technical stakeholders in the design process.

There is great need for re-using the modelling work done during the product lifecycle. Due to emerging modularity of simulation software (Figure 1), the re-using modelling work with virtual 
prototypes is gradually becoming possible. However, a high-fidelity product development simulator may need simplifications in order to achieve real-time simulation. Within this approach, the training simulator can be reached by combining physical systems (including real control) to the existing virtual simulator. Correspondingly, a digital twin can be achieved by combining the existing simulator and condition monitoring algorithms for real-time data stream by physical sensors.

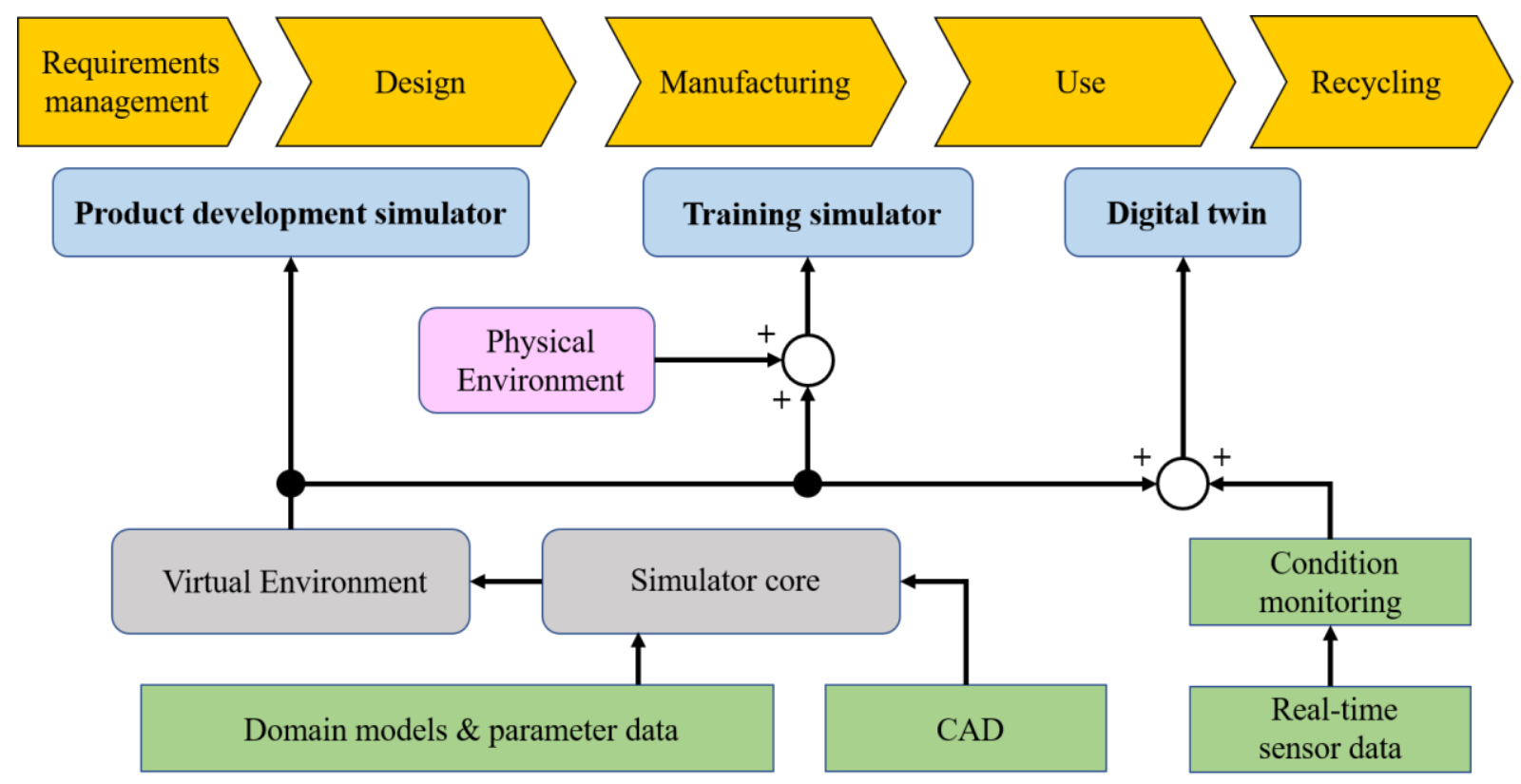

Figure 1. Overview of physics-based virtual prototypes for real-time simulation

\section{Framework from CAD to real-time simulation}

\subsection{Software in use}

\subsubsection{AGX Dynamics}

AGX Dynamics is a physics engine developed by Algoryx Simulation AB. It enables simulation in different fields of mechanics, materials, construction and industrial processes. Typical applications cover complex systems such as robots, vehicles and machines. This tool provides simulation-based design for testing and optimization, supports the development of training simulators and product demonstrations for marketing purposes (Servin and Brandl, 2018).

The software, built on original scientific research, descends from a discrete variational Lagrangian mechanics for constrained systems with dry frictional contacts. This makes it a powerful and robust simulation tool, providing physics-based results.

The simulations are composed by rigid bodies with geometry shapes and physical properties. Rigid bodies have a geometrical representation and a collision detecting mesh. Different geometrical constraints can be defined to create relations between rigid bodies and the space. The constraints are configurated in a form of joints that restricts their degrees of freedom (DOF), limiting translational and rotational freedom. Besides complete restriction, the joints enables, in the DOF not constrained, impose a certain traveling speed within a limited range. This allows to simulate behaviours in realtime with controlled variables or even with autonomous actions.

The physics engine calculates and integrates the simulation in a defined time-step. In each time-step it performs geometry overlap tests and contact collision detection. Contacts are detected and constantly solved during the simulation.

Simulations are built and managed directly through high level scripting. Other possibility is by using an integrated graphical user interface in other platforms as the CAD software SpaceClaim, through the external plug-in AGX Momentum, and the game engine Unity, through AGXUnity. 


\subsubsection{AGX Momentum}

AGX Momentum is the integration of the physics engine AGX Dynamics with SpaceClaim (3D parametric modelling CAD software) through a graphical user interface, enabling direct dynamic simulations of CAD models.

CAD software are typical tools used by designers and engineers to model complex and detailed systems. By combining AGX Momentum, static assemblies become physically dynamic, enabling immediate visual design impact. This has special emphasis in product development phase since it enables quick validation of design ideas directly in the process of CAD modelling. From an iterative process of model construction, input variables and dynamic simulations, undesirable behaviours are easily identified, and desirable behaviours may be enhanced.

This add-in generates a mesh tessellation from the modelled solids inside SpaceClaim and turns into AGX Dynamics simulation environment. The geometric constraints between bodies are manually set, enabling definition of behaviours and mechanical systems functions. This means that separately solids may be merged to act as a single body and interacting freely with other bodies, or even be coupled in certain way, limiting its DOF in the space.

Besides the fundamental assistance tool in design process, AGX Momentum enables the use of virtual prototypes for co-creation and marketing purposes. CAD models may be demonstrated in a dynamic way and in real-time, simplifying the communication between engineers and customers for model interaction, prompt modifications and demonstrations. This can be achieved by importing developed simulations into other platforms as Unity, through the external plug-in AGXUnity.

\subsubsection{Unity}

With the emergence of Head-Mounted Displays (HMDs) and computer graphic capabilities, powerful and flexible software tools became available to develop content in VR and AR. One popular example is the game engine Unity. This software makes possible to import very different type of format materials (as 3D models, shapes, images, videos, sounds, textures) that can be displaced in a scenario, add physic behaviours, controllers, interaction, lights, animation and generate a real-time simulator in a complete VE. This software supports development to different platforms. This means that human immersion and participation in virtual scenes built can occur through monitor displays (computers), portable smart displays (smartphones) or HMD devices (AR or VR devices).

Game engines as Unity have been used as assistance tools in different fields for research studies and industry applications. They are specially used in build hypothetical virtual scenarios. A few practical example cases are the development of machine simulators to train operators (Zheng et al., 2018), simulate and visualise multiple complex virtual scenes in collaborative design sessions (Hjelseth et al., 2015), support ideation of exterior and interior lighting in the early stages of product development (Ekströmer et al., 2019) or even construct Tangible AR product representations (Jain and Choi, 2019).

\subsubsection{AGXUnity}

Algoryx Simulation provides integration and support of the AGX Dynamics on Unity platform through AGXUnity. It enables access and use of the physics engine library scripts to construct simulations in real-time, affording real physical properties to solids and real collision solutions. Besides, it provides tools to simulate cables and motion behaviour of water and wind.

Simulations developed in AGX Momentum may be imported to Unity. All solids, constraints and simulation configurations previously built become available in this platform. Thus, CAD models are easily incorporated in VEs established in Unity. From here, real-time CAD simulators are achieved and may provide control interaction with real size prototype view through VR devices and physical controllers.

\subsection{Framework}

The software previously presented enable a connection between them. When combined, through different stages, they allow to explore, communicate and improve designs beyond what traditional CAD tools alone allow. 
By incorporating the AGX Momentum in the development of the CAD model in SpaceClaim, it enables directly construction of physics-based dynamic simulations.

Furthermore, this CAD simulation model can be exported through AGX Dynamics specific format file and direct imported to Unity through the AGXUnity plug-in. In Unity, VE may be developed to test the prototype.

The user participation in the virtual scene is established with the use of a VR device. Through physical devices as Oculus controllers, the user is enabled to interact with the model.

The prementioned stages form a framework that enables to achieve a real-time simulator from a CAD model using a robust physics engine. The user can control and interact with the prototype and perceives the real dimensions of the artefact. The Figure 2 represents the framework from CAD to real-time VR simulator.

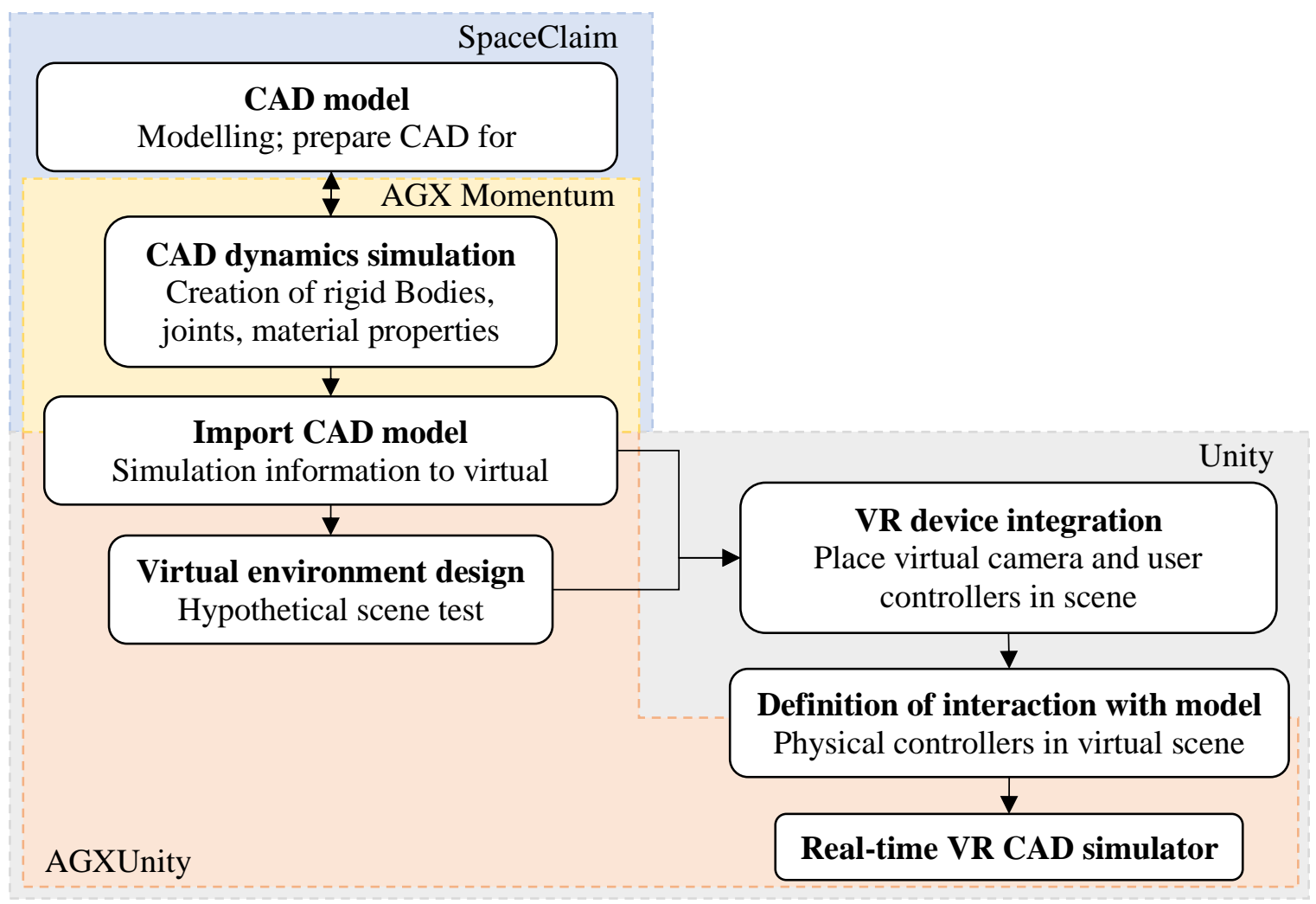

Figure 2. Framework from CAD to real-time simulator

\section{Case study: Harvester simulator}

\subsection{CAD model}

In order to study the applicability of the framework, a complex harvester was used in our case study (Figure 3). The harvester is Forest Master Turbo, developed by Usewood Forest Tec Oy. The company provided the harvester model in SolidWorks format to our research group. This is a good testing case since it is a complex and large model that can provide better understanding of the applicability of dynamics simulation tools and the factors that affect real-time simulation. Moreover, mobile machine prototyping usage with VR technology has been a research focus in the last decades, particularly in Finnish industry (Ellman and Tiainen, 2019), due to several fundamental factors involved in cabin prototyping design: ergonomics, visibility, functionality, safety and industrial design (Ellman et al., 2009; Tiainen et al., 2011).

The harvester CAD model consists of 3298 solids assembled in a main assembly file, representing individually each part of the machine and their static relations positions. Each component is modelled on real dimensions, representing the harvester on real scale. The machine has a length of 3,8 meters and a 
width of 1,5 meters. The prototype includes detailed bodies inside of rear and front frame (invisible from outside perspective), representing the cabin components, modelled engine and wheels transmission system. A portion of the model consists in small parts as screws, sealing rings and bearings.

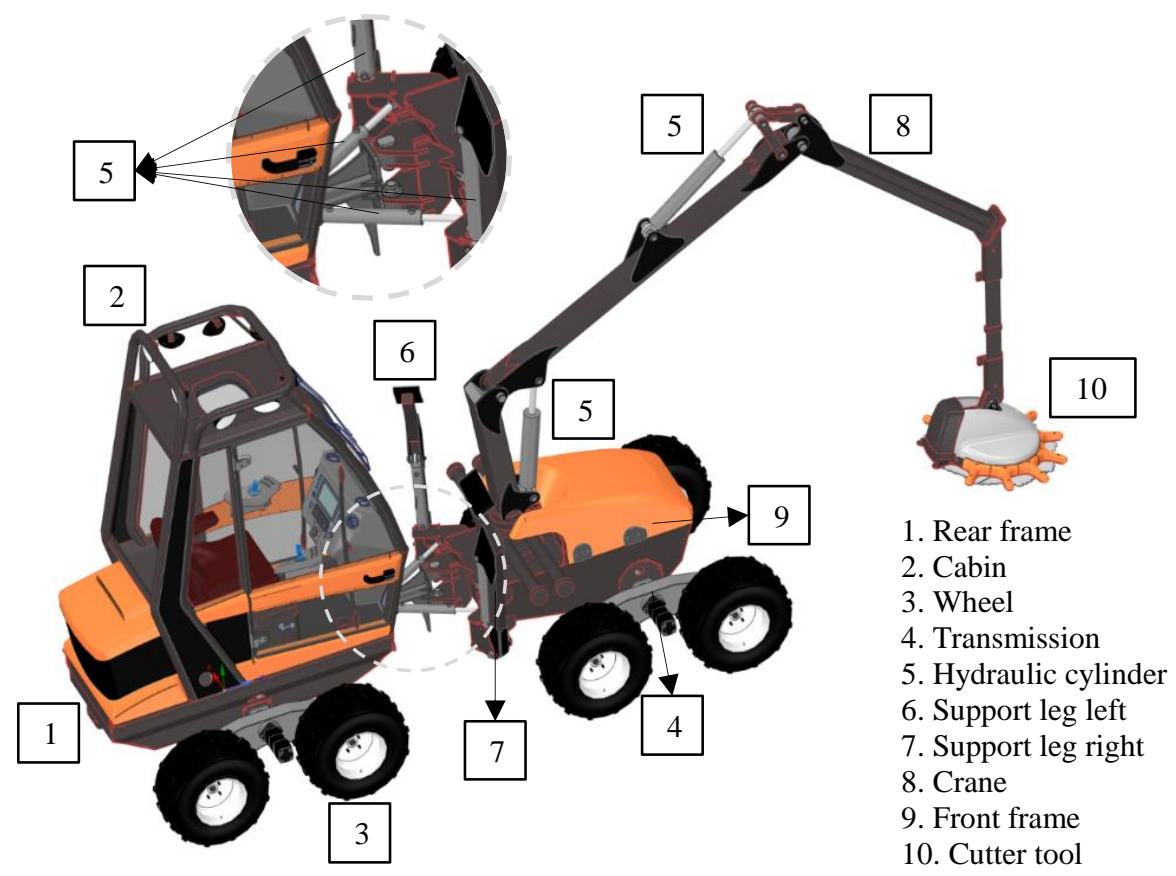

Figure 3. Harvester CAD model

The machine was designed to be controlled by human operator through the cabin interior. Here, the operator is provided with buttons, joysticks and a pedal to operate the different essential harvester components as the boom, the cutter tool attached to the boom, the support legs and the steering. The movements of these components are provided through hydraulic cylinders: the crane uses two hydraulic cylinders to lift and bend the arm; The cutter tool has an internal hydraulic cylinder connected to the crane; Both support legs have one hydraulic cylinder to control individual rotation in relation to the front frame; Two hydraulic cylinders are connected to rear and front frame to steer driving. The eight wheels in the machine are connected in pairs to transmission parts and these ones are separately coupled to the harvester frames.

The user must be able to control the components dynamics to drive the machine and control the support legs, crane and cutter tool. Another essential factor to implement in the simulator is related to the VE in use. Since this type of machine models manly operate in forest context, it makes sense to similarly provide the same environment to increase the reliability when running the simulator. For that reason, is intended to visualise and simulate the prototype in a virtual forest.

\subsection{Construction of the simulator}

The CAD model of the harvester was directly imported from SolidWorks to SpaceClaim. After an initial inspection it was decided that interior components (as engine) and numerous small solids in the model, such as screws, sealing rings and bearings were not considered to the simulation. However, their mass was not ignored, remaining to impact in the simulation. This decision was made to reduce the high number of components that do not need to be individually simulated without a great visual impact in the model, enhancing the simulation quality of the remaining components. This change reduced the model from 3296 to 527 solids.

The entire CAD dynamic simulation of the model was created using the AGX Momentum capabilities. Initially, bodies were added into main rigid bodies, taking into consideration their role in the machine dynamics. This means that fixed solids relations were combined as an entire and specific rigid body. All the pistons of the hydraulic cylinders were kept as individual rigid bodies of the external cylinder. In the end, the model was separated in 36 rigid bodies, namely 8 wheels, 6 hydraulic cylinders and 
individual pistons, 4 transmission parts, cabin, individual connection part (between rear and front frame), 2 support legs, front base, the crane with 5 different parts, the cutter tool and its separate cutter disk. Figure 3 shows all rigid bodies defined separated by colours.

Thereafter, it was established the relations between rigid bodies by limiting their DOF. This was done in the software by using the joints tool. It was used prismatic joints on pistons and cylinders in the hydraulic cylinders, enabling one translational DOF between them. To spin the wheels, hinge joints were used, enabling only one rotation DOF in relation to the transmission part. Similarly, the same type of joint was used in the remaining rigid bodies relations. A total of 41 joints were added to the model.

A few joints created (wheels and cutter hinges, hydraulic cylinders prismatic joints) were configurated with speed traveling on their DOF, enabling dynamic movement. This means that the wheels will rotate, and the hydraulic cylinders will travel directionally, each with a defined speed. The Figure 4 shows also the rigid bodies relations with prismatic (straight arrows) and hinge joints (curved arrows) represented.

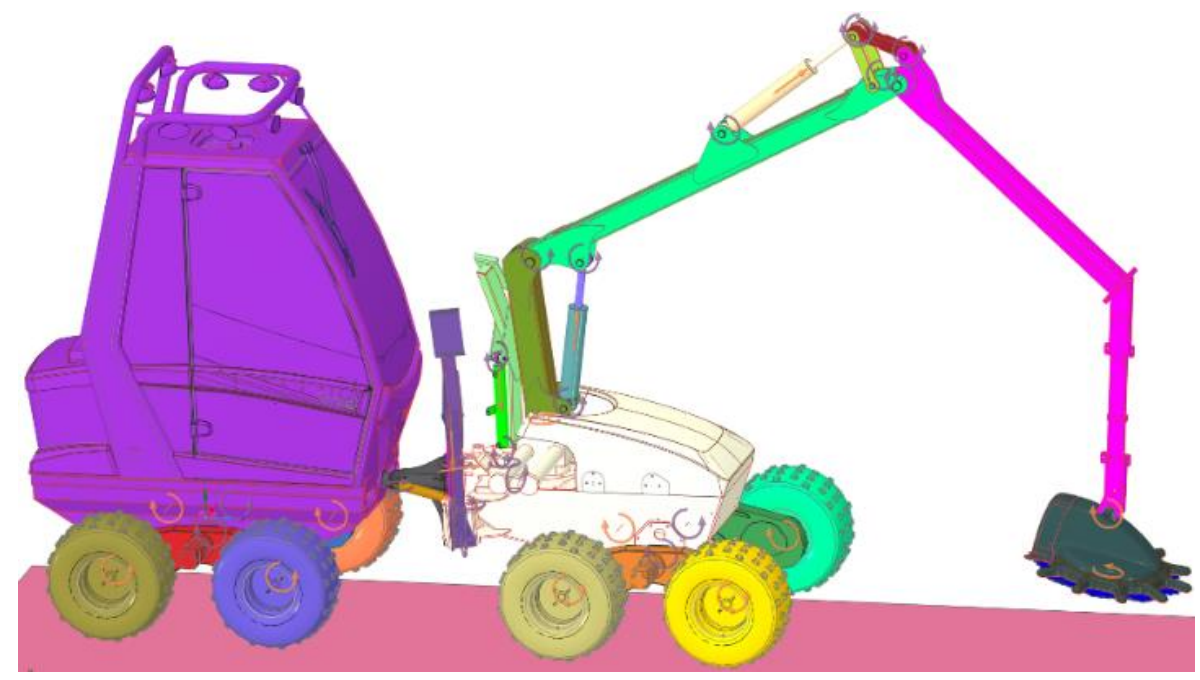

Figure 4. Rigid bodies and joints defined on CAD model

Different materials were added to the tires, disk cutter and support legs since these are the only components that create contact interactions with the ground. The CAD model was then simulated in AGX Momentum, observing its behaviour when landed in a flat surface (ground).

This simulation model was exported as AGX Dynamics file to a virtual forest scene, created in Unity. This forest is composed by a terrain with different height and textures, designed to pretend irregular and mountainous floor with realistic graphic visualisation. Different types and sizes of vegetation were also disposed arbitrarily.

The VR device Oculus Rift was integrated in the Unity scene, disposing the virtual camera inside the cabin component. A script was developed to permit interaction between the harvester model and the Oculus controllers, detecting buttons/joysticks input and changing the active joints speed from zero to a value previously defined. This enabled physical controllers interact with the CAD model in real-time.

The virtual simulator was built and tested with a desktop computer with an Intel processor i $72.67 \mathrm{~Hz}$, 6GB of memory RAM and a dedicated NVIDIA GTX 970 graphic card.

\subsection{VR experience of harvester simulator}

The simulator was tested in our office space (Figure 5), using a desk chair as initial user position, coinciding with user place on the scene, sitting in the cabin. The HMD provides the virtual visualization combined with the user head orientation and position in the real world. In the scene is possible to visualize the CAD model on its real size, with its modelled solids maintaining the same assembly relations, dimensions, shape and colour (Figure 5). The transparent cabin windows allow the observation of the forest and the machine components exactly from the driver viewpoint. 


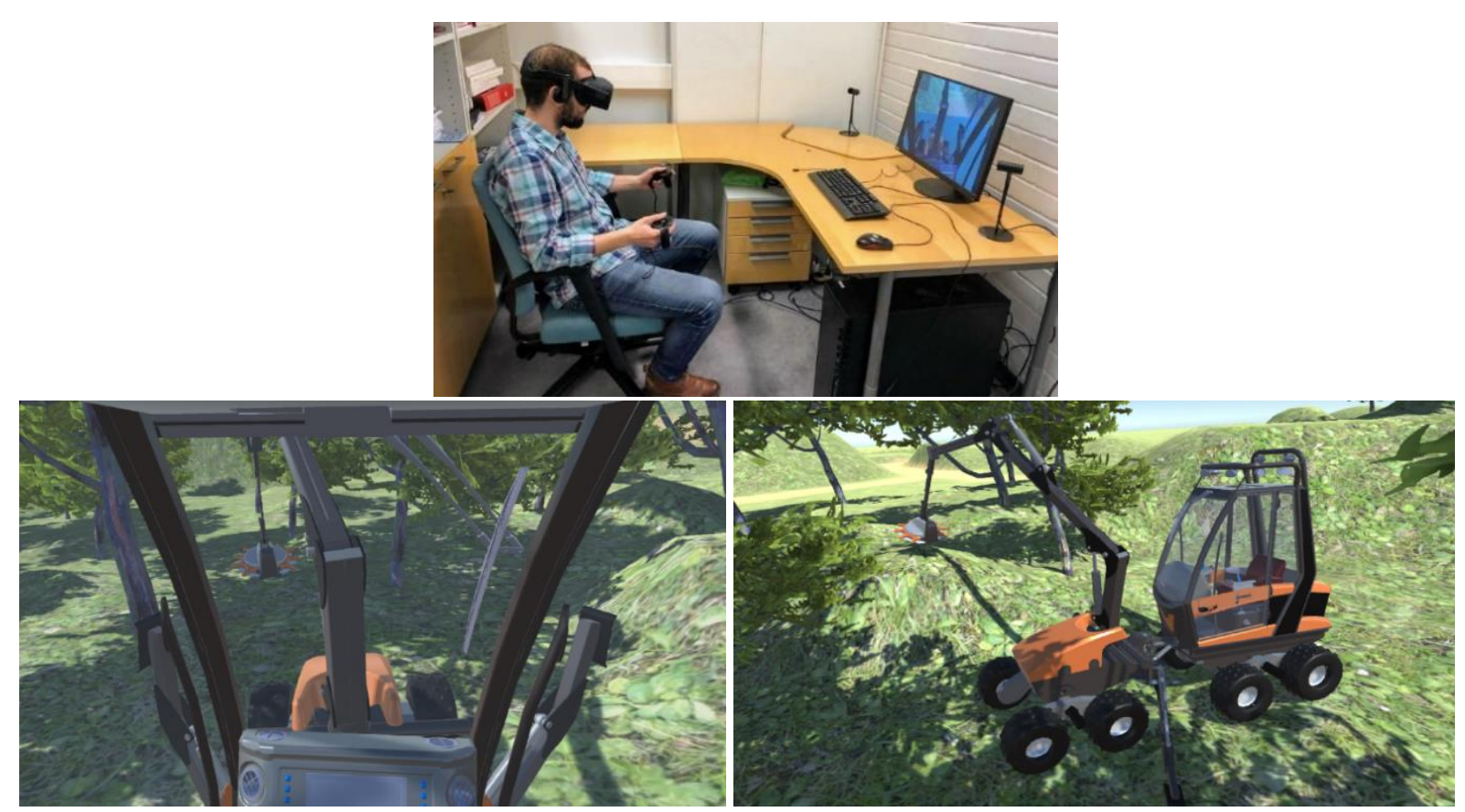

Figure 5. User testing simulator (top), VR perspective inside cabin (left) and CAD model in the virtual forest (right)

Through the Oculus controllers, is possible to interact with the components and consequently operate the machine in real-time. The user can control the wheels rotation direction, performing backwards and frontwards movements, and control the hydraulic cylinder prismatic position, controlling the steering driving and manipulating the crane and support legs.

The actions produced by the manipulation of the machine generates a realistic reaction in the virtual scene, giving a realistic and precise feedback to the cabin. This makes possible the user understands their own actions and the machine behaviour.

\subsection{Efforts analyses of the framework}

The proposed framework was applied on a harvester simulator. Table 1 resumes the tasks performed followed by an evaluation time.

Table 1. Time effort evaluation

\begin{tabular}{|c|c|c|c|c|c|c|}
\hline \multicolumn{3}{|c|}{ Software involved } & \multirow{3}{*}{$\begin{array}{c}\text { Framework steps } \\
\text { CAD model }\end{array}$} & \multirow[t]{2}{*}{ Main Tasks } & \multicolumn{2}{|c|}{ Time spent } \\
\hline & & & & & Hours & $\%$ \\
\hline$\Xi$ & & & & Import CAD model; prepare CAD to simulation & 2 & $13 \%$ \\
\hline $\begin{array}{l}0 \\
0 \\
\tilde{\Xi} \\
\text { के }\end{array}$ & \multirow{6}{*}{ 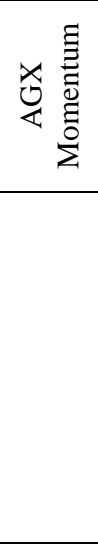 } & & $\begin{array}{l}\text { CAD dynamics } \\
\text { simulation }\end{array}$ & $\begin{array}{l}\text { Definition of rigid bodies, joints, material pairs; } \\
\text { iterative dynamic simulation and test }\end{array}$ & 5 & $31 \%$ \\
\hline \multirow{5}{*}{$\stackrel{\vec{\Xi}}{\Xi}$} & & \multirow{2}{*}{ 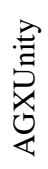 } & Import CAD model & Import CAD dynamics simulation material & 0.4 & $3 \%$ \\
\hline & & & $\begin{array}{l}\text { Virtual Environment } \\
\text { design }\end{array}$ & $\begin{array}{c}\text { Design the virtual forest with terrain, texture and } \\
\text { vegetation }\end{array}$ & 2 & $13 \%$ \\
\hline & & & VR device integration & $\begin{array}{l}\text { Usability of Oculus tools to integrate HMD and } \\
\text { physical controllers }\end{array}$ & 1.5 & $9 \%$ \\
\hline & & \multirow{2}{*}{ 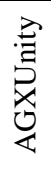 } & $\begin{array}{l}\text { Definition of user } \\
\text { machine control }\end{array}$ & $\begin{array}{l}\text { Script development for controller input and active } \\
\text { joint speed change effect }\end{array}$ & 3 & $19 \%$ \\
\hline & & & $\begin{array}{l}\text { Real-time CAD } \\
\text { simulator }\end{array}$ & Iterative changes and improvements & 2 & $13 \%$ \\
\hline & & & & Total time & \multicolumn{2}{|c|}{15.9 hours } \\
\hline
\end{tabular}


Initially, the work focused on importing the model from SolidWorks and disregarding very small components. The dynamic simulation essentially was based on the construction of the harvester model simulation by defining the bodies, configurate constraints and materials properties. Here, all dynamic relations and the control of the model were defined, being highly influential in the subsequent simulation.

The VE design in Unity appealed to assets already pre-configured, focusing on trying to generate a most realistic forest scenario. Importing the simulation model developed to this VE revealed to be a very simple task.

The incorporation of Oculus Rift into the scene was simplified by the already built integration package provided by Oculus. Enable interaction between user and model in real-time was established by scripting, taking some time due to physical control and software connection involvement. The last stage entailed an iteration between testing the simulator and configurations adjustments.

The usability of a physics engine in real-time provides high computation load. This affects not only the simulation itself but also the VR experience of the user. The initial simulator tests performed revealed a higher calculation time necessary for each time-step, leading to slow and unrealistic behaviour with poor graphics performance. Such heavy computational performance may be considered a natural consequence of the complex and huge model.

To reduce the calculations time, some simplifications were taken. The number of contact points were reduced by using hidden flat cylinder solids as wheels. Also, the contact parameter was disabled for components without interaction with other components, avoiding their constant overlap tests performance. With these configurations it was achieved a real-time simulator without constrained physic calculations, running at around 25 frames per second (fps). Table 2 resumes the model and final simulator information.

Table 2. Information resume of simulator achieved

\begin{tabular}{|c|c|c|c|c|c|c|}
\hline $\begin{array}{c}\text { Real-time } \\
\text { simulator }\end{array}$ & $\begin{array}{c}\text { Solids in the } \\
\text { model }\end{array}$ & $\begin{array}{c}\text { Rigid } \\
\text { bodies }\end{array}$ & $\begin{array}{c}\text { Rigid bodies with contact } \\
\text { enabled }\end{array}$ & Joints & Polygons & $\begin{array}{c}\text { Frame } \\
\text { rate }\end{array}$ \\
\hline $\begin{array}{c}\text { Harvester } \\
\text { CAD model }\end{array}$ & 527 & 36 & 11 & 40 & 545143 & $\sim 25 \mathrm{fps}$ \\
\hline
\end{tabular}

\section{Discussion}

The presented framework enabled to implement a physics-based virtual prototype from a CAD model. Constructing a physics-based simulator requires usually significant workload. However, by applying several modular software it was possible to achieve this with a meaningful workload and executive time. In our case study we showed that 3D CAD model can be implemented to a physics-based real-time VE simulator. This is a result of the different approach of this framework, where the AGX Momentum has an autonomous role in model conversion inside SpaceClaim. In consequence, merging CAD models to the game engine Unity is no longer a hard and complicated process. However, handling game engine platforms can make it difficult to implement other prototypes with the physical controllers or sensors. The use of physics-based virtual prototypes in VE, as our case study, can be seen from different angles on product lifecycle management.

From a product development perspective, it enables a profound understanding of the design and functional structure, with rapid identification of predictable and unpredictable behaviours. Furthermore, it enables new possibilities on design communication and co-creation due to model dynamic visualisation and high sense of realism.

In a teaching perspective, such simulators may have training application. Future users will be able to interact with the product, comprehend accurately hypothetical real use scenarios and avoid potential hazards in a short period of time after or even during its development phase.

In a digital twin perspective, it will further allow to monitor the product until end-of-life when combined with real-time data from physical sensors. This will provide the use of external information for further product models with a wide level of virtual simulation.

Although a simulator with a good level of realism was achieved, it was remarkable that the simulator showed some computer performance restrictions. The harvester used is a huge model, but there are other 
systems with a higher level of complexity and in future even more complex and elaborative will become. For future simulations, the hardware in use will be reviewed and higher performance should be used.

\section{Conclusions}

Our study proved how to transform a 3D CAD model to physics-based real-time prototype through the presented framework. The case study showed that this can be realized with a meaningful working effort. The framework used for implementation of physics-based virtual prototypes can be established for different phases of product lifecycle. It enables the use as a product development simulator, a training simulator and further as a digital twin. These outcomes play a crucial role in the product lifecycle management, contributing for efficiency and feedback for further product development activity.

\section{References}

Berg, L.P. and Vance, J.M. (2017), "Industry use of virtual reality in product design and manufacturing: a survey", Virtual Real, Vol. 21, pp. 1-17. https://doi.org/10.1007/s10055-016-0293-9

Ekströmer, P. et al. (2019), "Shedding Light on Game Engines and Virtual Reality for Design Ideation", Proceedings of the Design Society: International Conference on Engineering Design, Vol. 1 No. 1, pp. 2003-2010, Cambridge University Press. https://doi.org/10.1017/dsi.2019.206

Ellman, A. and Tiainen, T. (2019), "Diffusion of Innovation: Case of Co-Design of Cabins in Mobile Work Machine Industry", Computers, Vol. 2019 No. 8, pp. 39. https://doi.org/10.3390/computers8020039

Ellman, A. et al. (2018), "Evaluating a Virtual Wind Power Park in a Churchyard: A Perception Study With Portable VR Devices", Volume 1B: 38th Computers and Information in Engineering Conference, American Society of Mechanical Engineers, pp. 1-8. https://doi.org/10.1115/DETC2018-85752

Ellman, A. et al. (2009), "Combination of Virtual and Physical Objects in User-Centered Design of a Mobile Work Machine Cabin”, Proceedings of the ASME 2007 International Mechanical Engineering Congress and Exposition. Volume 3: Design and Manufacturing, Seattle, Washington, USA, November 11-15, 2007, ASME, pp. 143-148. https://doi.org/10.1115/IMECE2007-41778

Esqué, S. et al. (2003), "Techniques for Studying a Mobile Hydraulic Crane in Virtual Reality", Int. J. Fluid Power, Vol. 4, pp. 25-35. https://doi.org/10.1080/14399776.2003.10781163

Grieves, M.W. and Vickers, J. (2017), Digital Twin: Mitigating Unpredictable, Undesirable Emergent Behavior in Complex Systems, Springer International Publishing. https://doi.org/10.1007/978-3-319-38756-7_4

Hjelseth, S. et al. (2015), "Design and Computer Simulated User Scenarios: Exploring Real-Time 3D Game Engines and Simulation in the Maritime Sector", International Journal of Design, Vol. 9 No. 3, pp. 63-75.

Horvat, N. et al. (2019), "Comparing Virtual Reality and Desktop Interface for Reviewing 3D CAD Models", Proc. Des. Soc. Int. Conf. Eng. Des., Vol. 1, pp. 1923-1932. https://doi.org/10.1017/dsi.2019.198

Jain, K. and Choi, Y.M. (2019), "Building Tangible Augmented Reality Models for Use in Product Development", Proceedings of the Design Society: International Conference on Engineering Design, Vol. 1 No. 1, pp. 1913-1922, Cambridge University Press. https://doi.org/10.1017/dsi.2019.197

Lacoursière, C. (2007), Ghosts and machines: regularized variational methods for interactive simulations of multibodies with dry frictional contacts [PhD Thesis], Umeå University.

Servin, M. et al. (2018), "Physics-based virtual environments for autonomous earthmoving and mining machinery”, In: Berns, K. (Ed.), Commercial Vehicle Technology, Vol. 2018, pp. 493-504. https://doi.org/ 10.1007/978-3-658-21300-8_38

Tiainen, T. et al. (2011), "Three frames for studying users in virtual environments: Case of simulated mobile machines", Proceedings - IEEE International Symposium on Distributed Simulation and Real-Time Applications, Salford, 2011, pp. 115-119. https://doi.org/10.1109/DS-RT.2011.21

Tiainen, T. et al. (2014), "Virtual prototypes reveal more development ideas: comparison between customers' evaluation of virtual and physical prototypes", Virtual Phys. Prototyp, Vol. 9, pp. 169-180. https://doi.org/ $10.1080 / 17452759.2014 .934573$

Zheng, Y. et al. (2018), "Research on Virtual Driving System of a Forestry Logging Harvester", Wireless Personal Communications. https://doi.org/10.1007/s11277-017-5085-3 